\title{
UAV Robust Strategy Control Based on MAS
}

\author{
Jian Han, Changhong Wang, and Guoxing Yi \\ Research Center of Space Control and Inertial Technology, Harbin Institute of Technology, Harbin 150001, China \\ Correspondence should be addressed to Jian Han; jian.han.hit@gmail.com
}

Received 23 March 2014; Revised 13 April 2014; Accepted 13 April 2014; Published 5 May 2014

Academic Editor: Hamid Reza Karimi

Copyright (C) 2014 Jian Han et al. This is an open access article distributed under the Creative Commons Attribution License, which permits unrestricted use, distribution, and reproduction in any medium, provided the original work is properly cited.

\begin{abstract}
A novel multiagent system (MAS) has been proposed to integrate individual UAV (unmanned aerial vehicle) to form a UAV team which can accomplish complex missions with better efficiency and effect. The MAS based UAV team control is more able to conquer dynamic situations and enhance the performance of any single UAV. In this paper, the MAS proposed and established combines the reacting and thinking abilities to be an initiative and autonomous hybrid system which can solve missions involving coordinated flight and cooperative operation. The MAS uses BDI model to support its logical perception and to classify the different missions; then the missions will be allocated by utilizing auction mechanism after analyzing dynamic parameters. Prim potential algorithm, particle swarm algorithm, and reallocation mechanism are proposed to realize the rational decomposing and optimal allocation in order to reach the maximum profit. After simulation, the MAS has been proved to be able to promote the success ratio and raise the robustness, while realizing feasibility of coordinated flight and optimality of cooperative mission.
\end{abstract}

\section{Introduction}

UAV has stepped into the new generation and gained more powerful skills to accomplish a wide range of missions with high efficiency and high accuracy rate. At present, using UAV team to complete much more complex mission has become the most likely trend in modern war, but the performance of single UAV will be legged when executing coordinated flight and cooperative mission because of the limited resources it holds. The limited abilities will form the cask principle, and this will ultimately affect the effeteness and effect of mission execution. When it comes to the real battlefield, it will become more and more obvious due to the myriad changes in the circumstances and environment.

Utilizing MAS to improve the success rate and robustness in multiple UAV cooperation is a new research direction, because as a new generation of intelligent system, MAS has much more flexibilities $[1,2]$. It is more capable of solving the problems of coordination [3] and optimization and, moreover, has a very good adaptability for the uncertainties $[4,5]$ in the complex system. More than accepting tasks in a passive way, agent can also take the initiative to request tasks in an active way by sharing and exchanging with other agents to complete coordination, consultation, and collaboration
$[6,7]$, which makes MAS not just a form of collection but an agent organism to strengthen the robustness of the UAV team. The MAS can well adapt to the complicated and distributed task forms [8] and reduce human involvements in decision making process which will greatly ensure the timely and successful completion of tasks.

The MAS with BDI model [9] gives every individual intelligent algorithms, with which the UAV can figure out the cooperative control solution according to its state and task target automatically and then complete the trajectory tracking, formation changing, obstacle avoidance, and cooperative task processing [10]. Agent can acquire useful information from others according to its needs and then use the UAV mission allocation system based on auction mechanism [11, 12] to solve the coupled task allocation problem and dynamic reallocation problem.

\section{Establish the Multiagent System}

The MAS regards every UAV as a control subject. In order to realize coordinated flight, cooperative mission and raise the robustness of MAS, tracking mechanism, the artificial potential field (APF) mechanism, and the auction mechanism 


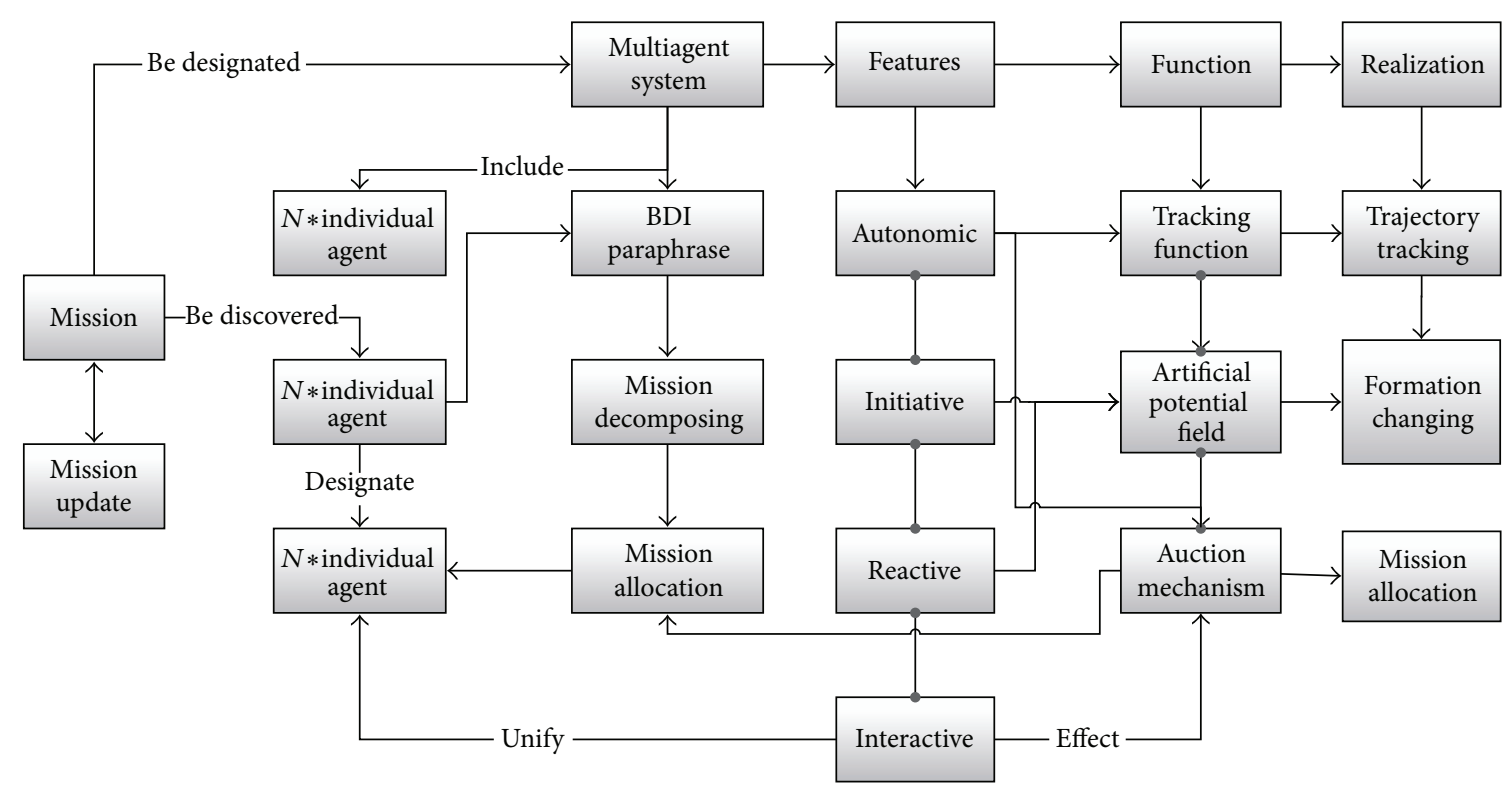

FIgURE 1: Structure of the cooperative system based on agent.

are established in the system. The specific structure is shown in Figure 1.

\subsection{Foundational Abilities: Reaction}

2.1.1. Tracking. Using analytic method or numerical method to guide UAV to follow a trajectory cannot match the requirement of timeliness, so the tracking mechanism utilizing approximation without establishing differential equations shows its convenience in real situation. At the time of $t=\tau$, UAV locates at $P_{1}\left(x_{1}, y_{1}\right)$, where $x_{1}=0, y_{1}=V_{U} \tau$, and target is at $M_{1}\left(V_{T} \tau, H\right)$. UAV begins with the direction of $P_{1} M_{1}$, and the slope angle of $P_{1} M_{1}$ is $\theta_{1}=\arctan \left(H-V_{U} \tau\right) / V_{T} \tau$, as shown in Figure 2. Following this way, as specific as in

$$
\begin{gathered}
x_{k+1}=x_{k}+V_{U} \tau \cos \theta_{k}, \\
y_{k+1}=y_{k}+V_{U} \tau \sin \theta_{k}, \\
\cos \theta_{k}=\frac{k V_{T} \tau-x_{k}}{\sqrt{\left(k V_{T} \tau-x_{k}\right)^{2}+\left(H-y_{k}\right)^{2}}}, \\
\sin \theta_{k}=\frac{H-y_{k}}{\sqrt{\left(k V_{T} \tau-x_{k}\right)^{2}+\left(H-y_{k}\right)^{2}}},
\end{gathered}
$$

the MAS will calculate the position deviation between UAV and target to plan the optimal trajectory in a short time to realize the real-time tracking.

2.1.2. Formation. APF (artificial potential field) is proposed to keep the formation of UAV team. The potential force $U_{i j}$ which is derived from the relative distance expresses the

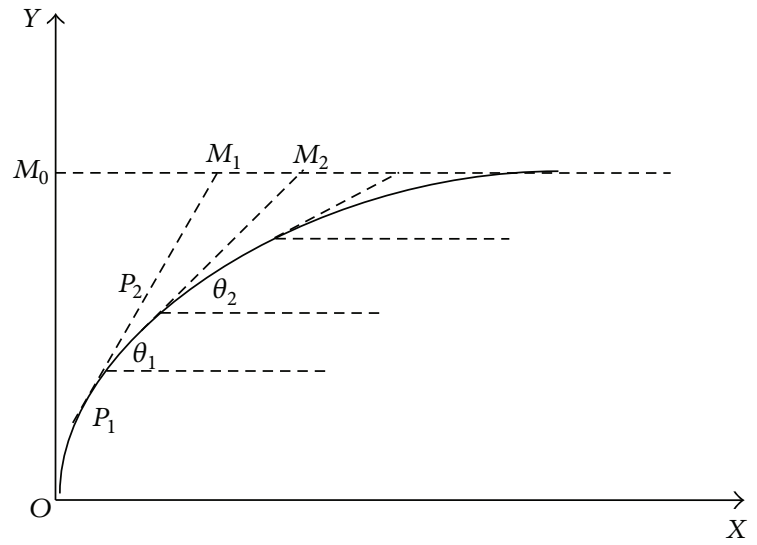

FIGURE 2: Target tracking by UAV.

potential field of relative distance $\left\|r_{i j}\right\|$, so the total potential among UAV team can be expressed as

$$
\begin{aligned}
U & =\sum_{j \in N_{i}} U_{i j}\left(\left\|r_{i j}\right\|\right) \\
& =\chi_{N_{0}}(i) \cdot U_{i 0}\left(\left\|r_{i 0}\right\|\right)+\sum_{j \in N_{i} /\{0\}} U_{i j}\left(\left\|r_{i j}\right\|\right) .
\end{aligned}
$$

$U_{i 0}\left(\left\|r_{i 0}\right\|\right)$ represents the potentialbetween the UAV and the target, $\sum_{j \in N_{i} /\{0\}} U_{i j}\left(\left\|r_{i j}\right\|\right)$ represents the total potential between one UAV and others, and $\chi_{N_{0}}(i)$ represents the weight parameter which is effected by potential formation samples stored in the library, as shown in Table 1. These samples can be updated during the formation changing in real-time. The potential field function will lead the UAV team to accomplish the position changing and make the process simpler in reality. 
TABLE 1: Formation example form.

\begin{tabular}{lccc}
\hline UAV $_{i}$ Position & $X_{\text {Left }}$ & $X_{\text {Center }}$ & $X_{\text {Right }}$ \\
\hline$Y_{\text {Front }}$ & 1 & 2 & 1 \\
$Y_{\text {Center }}$ & 2 & 0 & 2 \\
$Y_{\text {Back }}$ & 1 & 2 & 1 \\
\hline
\end{tabular}

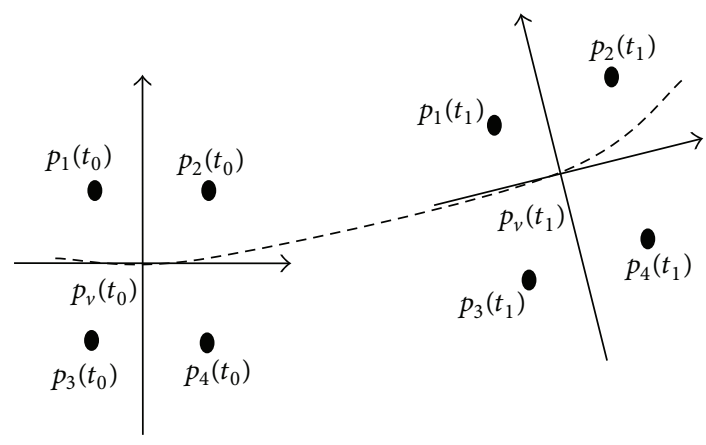

FIGURE 3: Structure of the center point.

2.1.3. Obstacle Avoiding. When a UAV team encounters an obstacle, the predetermined trajectory and the shape of the formation have to be abandoned. The shape of the formation is usually composed of irregular graphics, so an extra reference point representing the center of the formation is established to determine the overall trend of the UAV team. This virtual point is set to follow the predetermined mission trajectory and then help the UAV team to reorganize. The position of the center point $p_{v}(t)$ can be confirmed by relative positions of UAV around, expressed as $p_{v}(t)=\mathcal{Y}\left(\sum_{i=1}^{k} p_{i}(t)\right)$. The structure of the center point is shown in Figure 3 .

By introducing a gyration force $F_{i}^{g}=\omega(n, v) v$, the minimum problem with local dilemma has been resolved. The direction of the gyration force keeps being perpendicular to the speed in order to ensure the stability of the control system. The system will detect obstacles at any time; if any obstacle is on the way of motion or if the direction of obstacle avoidance is opposite to the speed, then the system will generate appropriate gyration force. This improved APF is as

$$
u_{i}=k F_{i}^{C A}+F_{i}^{d}+F_{i}^{V S}+k(r) F_{i}^{O A}+F_{i}^{g} .
$$

Forces in the above equation are set as follows. Repulsion force $F_{i}{ }^{\mathrm{A}}$ works for the avoidance motion. The stable position of any individual UAV in the formation is ensured by synchronization force $F_{i}^{d}$ and potential force $F_{i}^{C A}$ and, at the same time, they can complete the divergence and reorganization of the formation. Central attractive force $F_{i}^{V S}$ is used to make sure that any individual in the formation can follow the virtual point effectively.

2.2. Establishment of the BDI Model. Hybrid MAS combining reaction agent and thinking agent gives the system a better responsiveness and optimal planning ability. The structure of hybrid MAS is shown in Figure 4.

BDI model is used to describe the mission information in MAS, which is composed of three perceptions: belief (B), desire (D), and intention (I). Belief expressed as $\psi$ represents the motivation of processing missions which are generated after considering abilities of itself, restrictive conditions, and the environment around. In this MAS, the belief refers to abilities of both physical system and agent algorithms, which generally includes the capabilities of motion, navigation and location gained from the onboard equipments, and the capabilities of calculation, processing and communication gained from agent itself. Desire expressed as $\phi$ represents the eagerness of acquiring missions which can help the system gain benefits. The desire demonstrates expected results, such as the right to execute specific mission. Intention expressed as $\omega$ represents the method of solving mission in desire list. If $\omega$ cannot handle a mission, the system will consult with other agents to improve it, like when and how to make the formation changed; request information example is shown below:

$$
\begin{aligned}
& \text { @ Agent }{ }_{i}, \text { Problem }_{j}, \text { Time }_{k} \text {, Constaint }{ }_{l} \\
& \text { @ } \text { Agent }_{1} \text {, Monitoring Target } 1,10: 30 \text {, } \\
& \text { Accomplishment in } 2 \text { minutes } \\
& \text { @01,01 01,0A 1E, } 030078
\end{aligned}
$$

\section{Mission Allocation}

Individual agent may have certain otherness about the current overall world cognition which can be eliminated by sharing information and communication. The reasonable, prompt communication and interaction directly affect the success rate of mission processing, so the mission system has been devised in order to keep the allocation working smoothly, as shown in Figure 5.

3.1. Auction Mechanism. Auction mechanism [13] used in MAS is especially suitable for resource allocation problems with uncertainties. When a mission is released, every agent holding interests in it will bid for it. The bidder will decide its bidding according to the changing benefit, and the mission promulgator will give the execution right to the agent which makes the promulgator gain most benefit. The MAS auction process can be simply divided into four stages: mission releasing, mission bidding, winner deciding, and mission executing, as shown in Figure 6.

In this MAS, deadline mechanism, allocation confirming mechanism, and scale controlling mechanism are established to restrict auction scale. These can ensure the real-time, prevent the resource wasted by the bidder terminating the contract when the benefit changes, and also prevent bringing too much pressure to the communication and calculating if too many agents are involved.

3.2. Allocation of the Loosely Coupled Mission. The entire profit of the loosely coupled allocation is not only affected by the maximum benefit of every individual agent, but also more affected by the order of execution. It will greatly reduce the reasonableness of the allocation and be less efficient and optimal, in case of allocating missions without considering the relevance among them. So Prim-APF composed of 


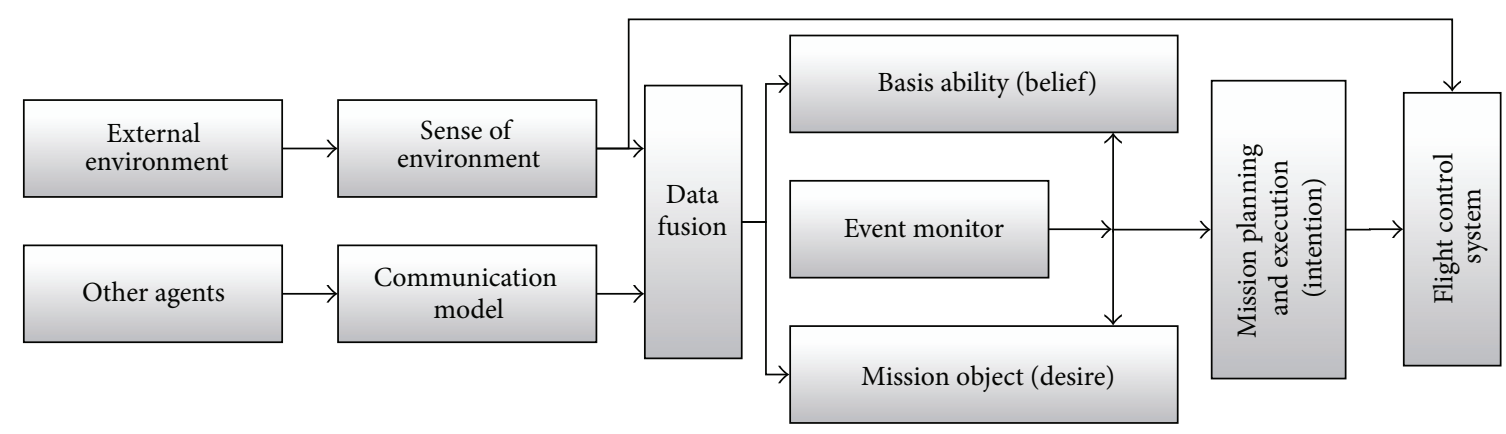

FIgURE 4: Structure of the hybrid system.

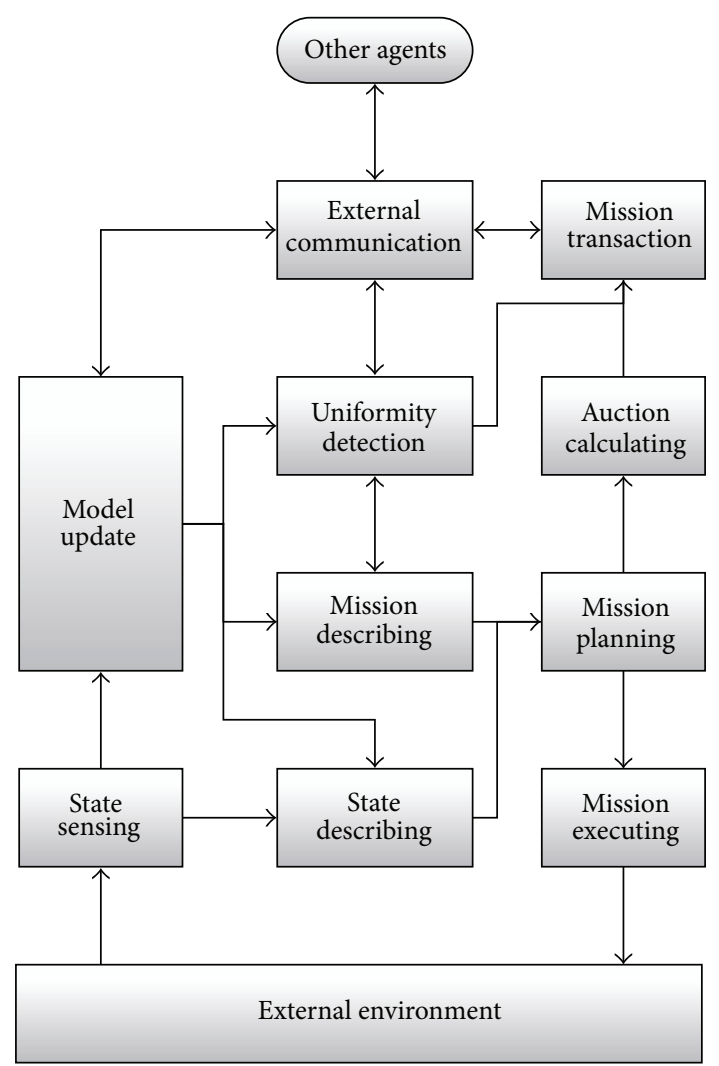

FIGURE 5: Float of the mission system.

Prim (namely, minimal spanning tree planning) and APF is devised to solve this optimal problem. This algorithm can decompose overall missions into small coordinate groups and then give each group a reasonable execution order to maximize the optimality.

For a mission of targets striking, if the missions are distributed in the original order without considering the relevance, it costs too much of 1313. When using ACO (ant colony optimization) to rearrange the order of the distribution, the global cost is decreased to 426 , but the ACO is not suitable for the actual operation because of the

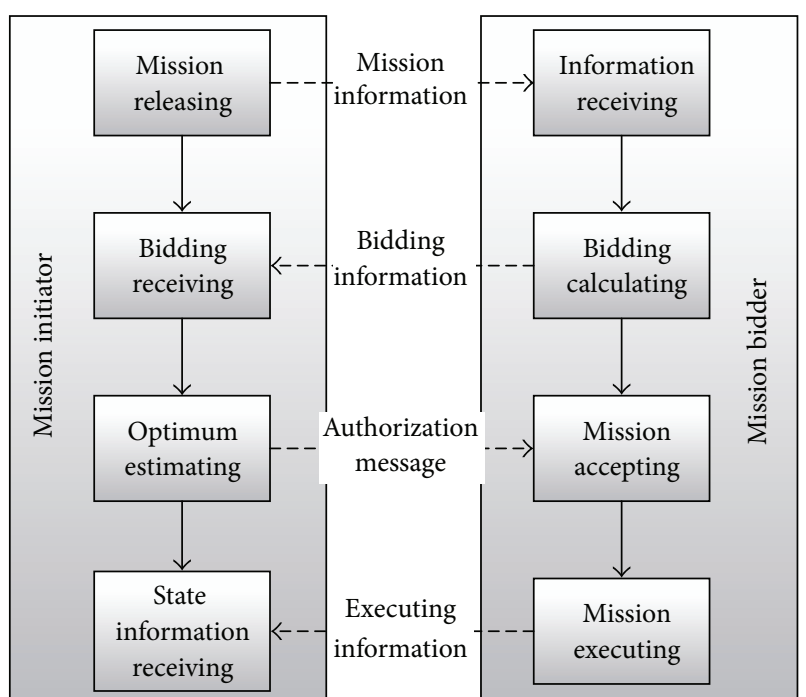

FIgURE 6: Float of the auction.

limitation and the time of calculation. The Prim-APF algorithm can do effective grouping with relevance before distributing, so the winner agent will get a group of missions with connections to ensure the optimality and improve the real-time. Using this algorithm, first, the entire group should be processed by Prim, as shown in Figure 7 .

Use the improved APF algorithm to calculate attraction force and repulsion force according to the target location and its branch, and then set weight parameters and valid paths to complete the optimal minimal envelope, as shown in Figure 8.

The result shows that Prim-APF (costs 438) is able to finish the allocation perfectly and saves more resources and improves the real-time due to its simpler computing, so it is better for actual missions which value time most. Considering the adaptability of the mission changing, this method will do minimal tree immediately for the remaining missions and has the lowest cost increasing; in the worst scenario, it will just increase the cost as much as the ACO.

3.3. Allocation of the Tightly Coupled Mission. As a cause of limitation, sometimes, accomplishing a mission needs agents 


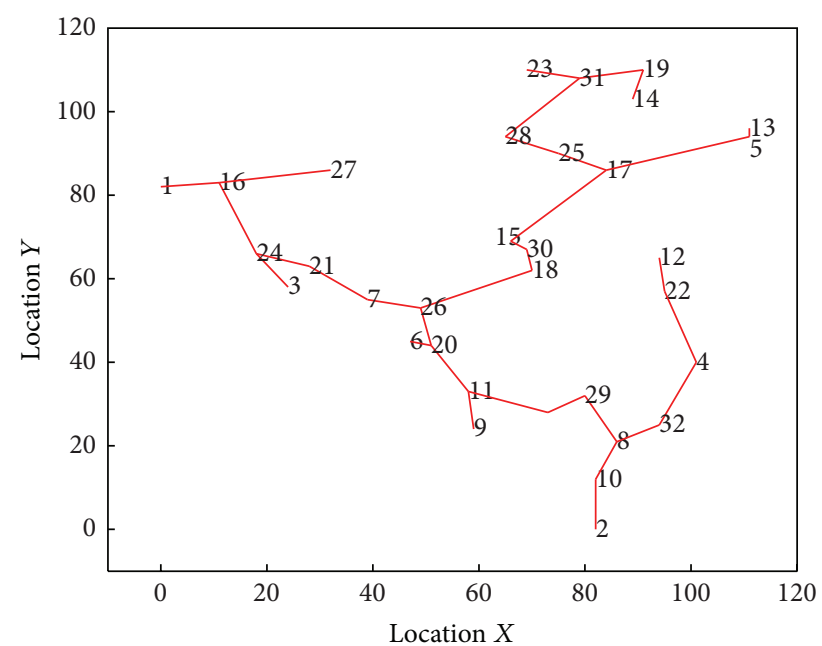

Figure 7: Output of the Prim algorithm.

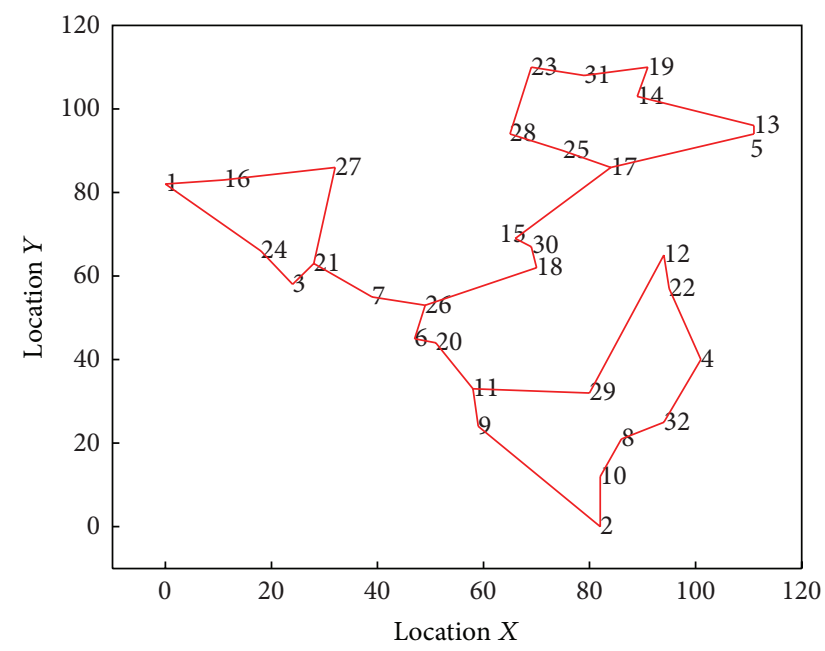

FIgUre 8: Output of the Prim artificial potential method.

coordinating in a logic order rather than only one agent, such as blockading some area. So an agent team mechanism is introduced to solve complex and difficult tightly coupled missions which can be simply divided into two kinds: one needs agents in the MAS to process the data parallel, such as joint monitoring; and the other needs agents to strictly obey the spatial order and chronological order, such as striking after scouting [14-16].

First, establish a matrix to represent the overall capabilities of the agent team, as in

$$
C^{C}\left(C_{k}\right)=\left[\begin{array}{cccc}
\alpha_{k 1}^{c} & 0 & \cdots & 0 \\
\cdots & \alpha_{k 2}^{c} & \cdots & 0 \\
\cdots & \cdots & \cdots & \cdots \\
0 & 0 & \cdots & \alpha_{k p}^{c}
\end{array}\right]\left[\begin{array}{c}
c_{1} \\
c_{2} \\
\vdots \\
c_{p}
\end{array}\right]
$$

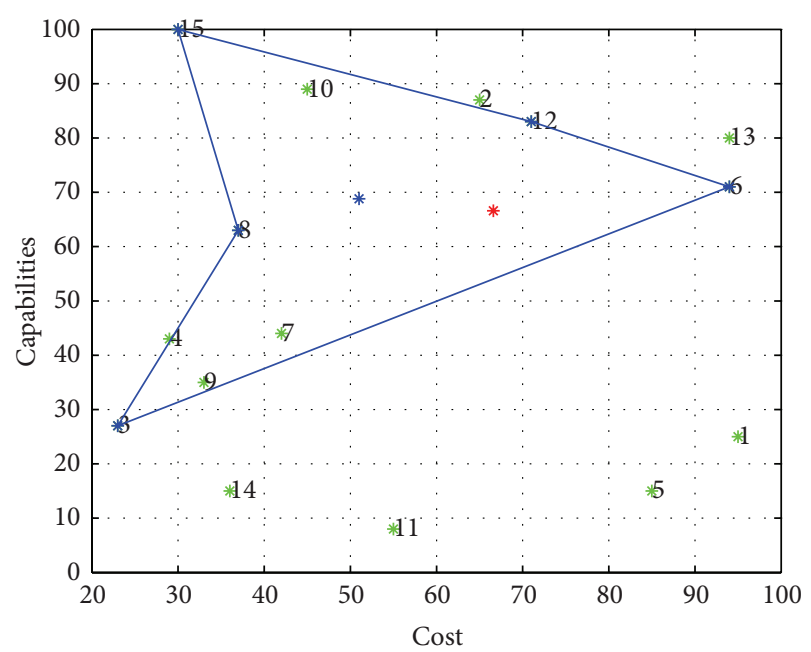

FIGURE 9: Result of the team selection.

The residual capabilities, expressed as $\Delta C^{c t}=\left(\delta_{1} c_{1}, \ldots\right.$, $\delta_{p} c_{p}$ ), should be minimized on the basis of mission constraints. The entire cost of executing the mission should be minimum, as in

$$
E_{\text {cost }}(C, t)=\sum_{i=1}^{q} E_{\text {cost }}\left(a_{i}, t\right)+C_{\text {cost }}(C, t),
$$

and the fault-tolerant capability of the team should be maximum, as in

$$
F_{\text {tolerant }}=\frac{w_{1} \times u_{1} \times u_{2} \times \cdots \times u_{q}}{(v / a)^{q}}+w_{2} \times\left(1-e^{-\lambda n}\right) .
$$

Different agent teams complete missions with different costs, so finding the most suitable team is significant but also will make the optimal problem harder. Using PSO (particle swarm optimization) to decide a suitable team is the best reply to complex structure and massive calculation. When selecting the team, the team capabilities should satisfy the mission requirements and always keep remaining capabilities and execution costs lower. A solution from simulation done by the PSO algorithm is shown in Figure 9; the blue represents the selected team capabilities and the red one represents the demanded capabilities.

In the PSO processing, give priorities to weigh parameters, fulfilling requirements better than execution cost and better than the remaining capabilities. These priorities will affect the particles, and the calculated fitness value of particle is proportional to the fine level of the team. After finding the team, send confirmed information to all agent team members; only if all these members have accepted the mission, the allocation information will be sent to them later; otherwise, the system will try to find other agents whose capabilities are close to the refused ones or begin a new team selection circle.

3.4. Allocation of the Dynamic Mission. A reasonable redistribution will make the MAS adapt to the circumstances 


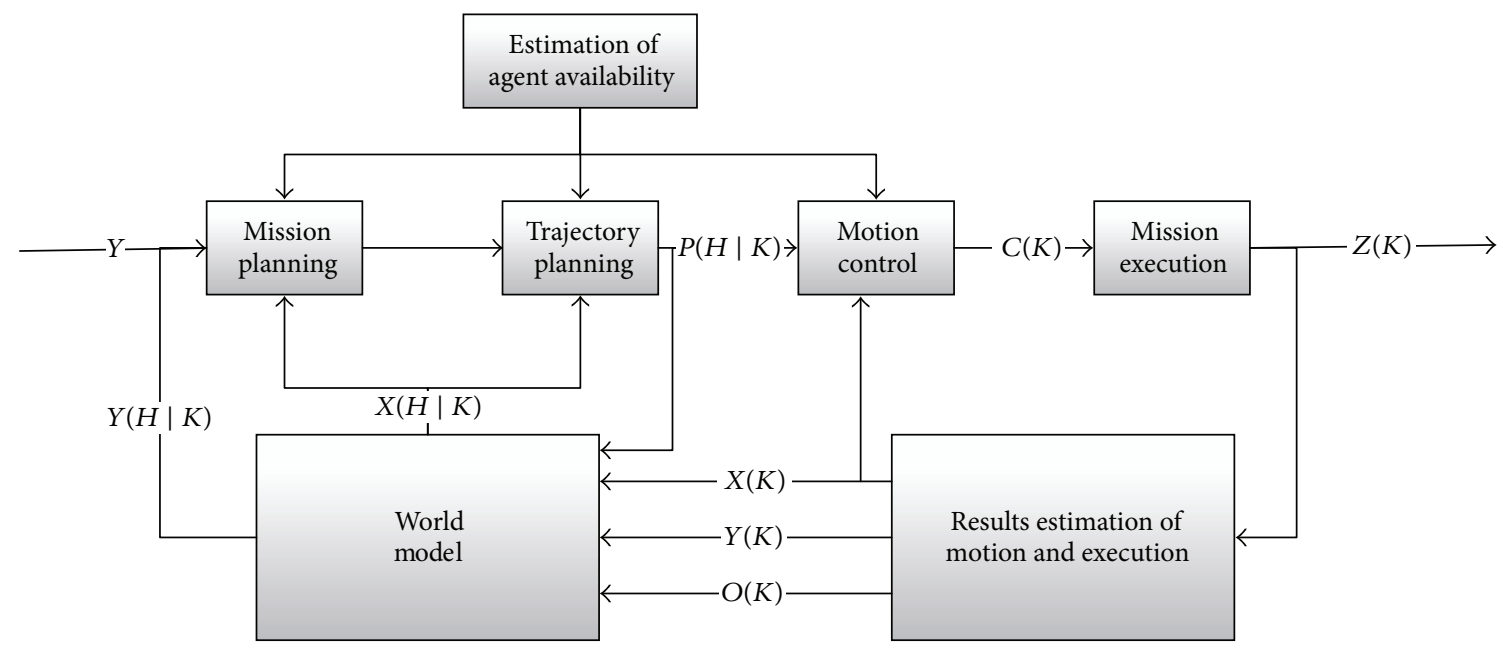

FIGURE 10: Control system of the mission redistribution.

of frequent mission changing, which will not only raise the resource utilization but also improve the computational efficiency. The redistribution control system is shown in Figure 10.

In this system, $Y$ as the initial solving command responds to the mission changing, and then it will be simulated and calculated to provide the solutions based on the availabilities of the current agents. In order to let $Y$ be more suitable for the current agent group, the solutions will be judged by the estimated cost changing, and then $Y$ will be updated via world model. This feedback will help the system to adapt to the mission changing and meanwhile prevent the overredistribution and the maldistribution. The auction algorithm is given as follows.

Algorithm. Willingness based redistribution.

Step 1. Calculate the cost of the remaining-mission redistribution, according to

$$
\begin{aligned}
E\left(t_{j}, h \mid k\right)= & \sum_{h=1}^{n} \alpha_{i}(h) \operatorname{Rew}_{j}\left(a_{i}, t_{j}, h \mid k\right) \\
& -\sum_{h=1}^{n} \beta_{i}(h) \operatorname{Cos}_{j}\left(a_{i}, t_{j}, h \mid k\right) .
\end{aligned}
$$

Step 2. Calculate the agent $a_{i}$ selling willingness value of the mission $t_{j}$ waiting redistribution, according to

$$
P_{\text {Agt }}\left(a_{i}, t_{j}\right)= \begin{cases}\frac{\left|D\left(a_{i}, t_{j}\right)\right|}{\sum_{D\left(a_{i}, t_{j}\right)<0}\left|D\left(a_{i}, t_{j}\right)\right|} & D\left(a_{i}, t_{j}\right)<0 \\ 0 & D\left(a_{i}, t_{j}\right) \geq 0 .\end{cases}
$$

Step 3. Form the willingness sequence Task_Sell for the mission being sold.

Step 4. Solve loosely coupled missions in Task_Sell which can be performed by one agent.
Step 5. Solve tightly coupled missions in Task_Sell which needs multiple agents to coordinate.

Step 6. Use the dynamic auction method to redistribute till Task_Sell is empty, according to the bidding willingness as in

$$
D\left(a_{i}, t_{j}\right)=E_{i}\left(t_{i}, h \mid k\right)-E_{i}\left(t_{j}\right) .
$$

\section{Conclusion}

In order to enhance the performance and robustness of coordination and cooperation in UAV team, hybrid MAS has been proposed with these novel algorithms: tracking mechanism in coordination flight, APF improved by model in formation changing, and improved APF in obstacle avoidance. An effective auction mechanism is devised to make MAS be able to respond to all kinds of mission, followed by the corresponding allocation algorithm, auction algorithm, and bidding algorithm. For loosely coupled missions, a Prim-APF algorithm of grouping allocation is designed. For tightly coupled missions, a PSO algorithm for selecting the best multiagent team is designed. For dynamic mission problems, a feedback control system based on the willingness is designed. These algorithms can achieve the optimal mission achievement and improve the robustness of the MAS.

\section{Conflict of Interests}

The authors declare that there is no conflict of interests regarding the publication of this paper.

\section{References}

[1] C. S. Mei, "Research and analysis of agent technology," Electronic Design Engineering, vol. 19, no. 11, pp. 121-124, 2011.

[2] J. Qiu, G. Feng, and J. Yang, "A new design of delay-dependent robust filtering for discrete-time T-S fuzzy systems with timevarying delay," IEEE Transactions on Fuzzy Systems, vol. 17, no. 5, pp. 1044-1058, 2009. 
[3] H. D. Han, Y. X. Wu, and Y. W. Cen, "Research progress of multirobot cooperation and coordination," Computer Engineering and Applications, vol. 44, no. 24, pp. 238-241, 2008.

[4] H. R. Karimi, "A computational method for optimal control problem of time-varying state-delayed systems by Haar wavelets," International Journal of Computer Mathematics, vol. 83, no. 2, pp. 235-246, 2006.

[5] S. Yin, H. Luo, and S. Ding, "Real-time implementation of faulttolerant control systems with performance optimization," IEEE Transactions on Industrial Electronics, vol. 64, no. 5, pp. 24022411, 2014.

[6] S. Yin, X. Yang, and H. R. Karimi, "Data-driven adaptive observer for fault diagnosis," Mathematical Problems in Engineering, vol. 2012, Article ID 832836, 21 pages, 2012.

[7] W. Lu, W. Zhiliang, H. Siquan, and L. Lei, "Ant colony optimization for task allocation in multi-agent systems," Communications, vol. 10, no. 3, pp. 125-132, 2012.

[8] W. D. Chen, Y. G. Xi, D. L. Gu, and S. L. Dong, "Complex task oriented multi-robot distributed coordination system," Control Theory and Applications, vol. 19, no. 4, pp. 505-510, 2002.

[9] W. H. Yi, H. G. Xia, and X. G. Chen, "Distributed coordination reasoning with BDI and knowledge level," Systems Engineering, vol. 22, no. 7, pp. 93-98, 2004.

[10] Z. Hui, Y. Shi, and M. Liu, "H step tracking control for networked discrete-time nonlinear systems with integral and predictive actions," IEEE Transactions on Industrial Informatics, vol. 9, no. 1, pp. 337-345, 2013.

[11] G. F. Tang and D. L. Jiang, "Study on task allocation and negotiation of military virtual warehouse multi-agent system based on auction theory," Logistics Technology, vol. 29, no. 12, pp. 193196, 2010.

[12] H. Klabi, K. Mellouli, S. Mellouli, and M. Rekik, "A trust model for a multi-agent negotiation," in Proceedings of the International Conference on Communications and Information Technology (ICCIT '12), pp. 291-296, June 2012.

[13] S. K. Moon, J. Park, T. W. Simpson, and S. R. T. Kumara, "A dynamic multiagent system based on a negotiation mechanism for product family design," IEEE Transactions on Automation Science and Engineering, vol. 5, no. 2, pp. 234-244, 2008.

[14] K. Osipov and G. Sukthankar, "Forming effective teams from agents with diverse skill sets," in Proceedings of the International Conference on Social Informatics (SocialInformatics '12), pp. 4448, December 2012.

[15] P. Dasgupta, "Multi-agent coordination techniques for multirobot task allocation and multi-robot area coverage," in Proceedings of the International Conference on Collaboration Technologies and Systems (CTS '12), p. 75, May 2012.

[16] X. Liu and D. Wang, "The behavior analysis of grouped multiattribute auction based on multi-agent.," in Proceedings of the 24th Chinese Control and Decision Conference (CCDC '12), pp. 2058-2062, May 2012. 


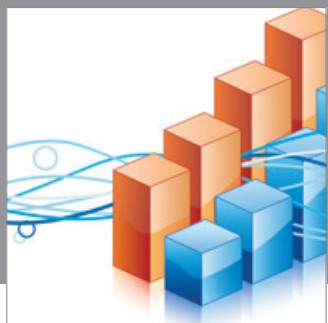

Advances in

Operations Research

mansans

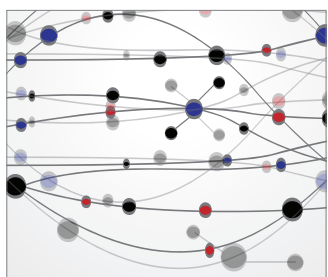

The Scientific World Journal
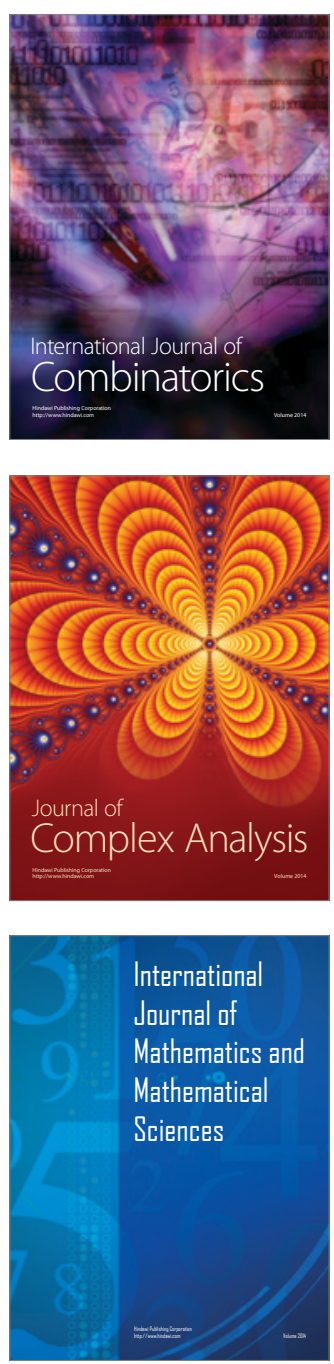
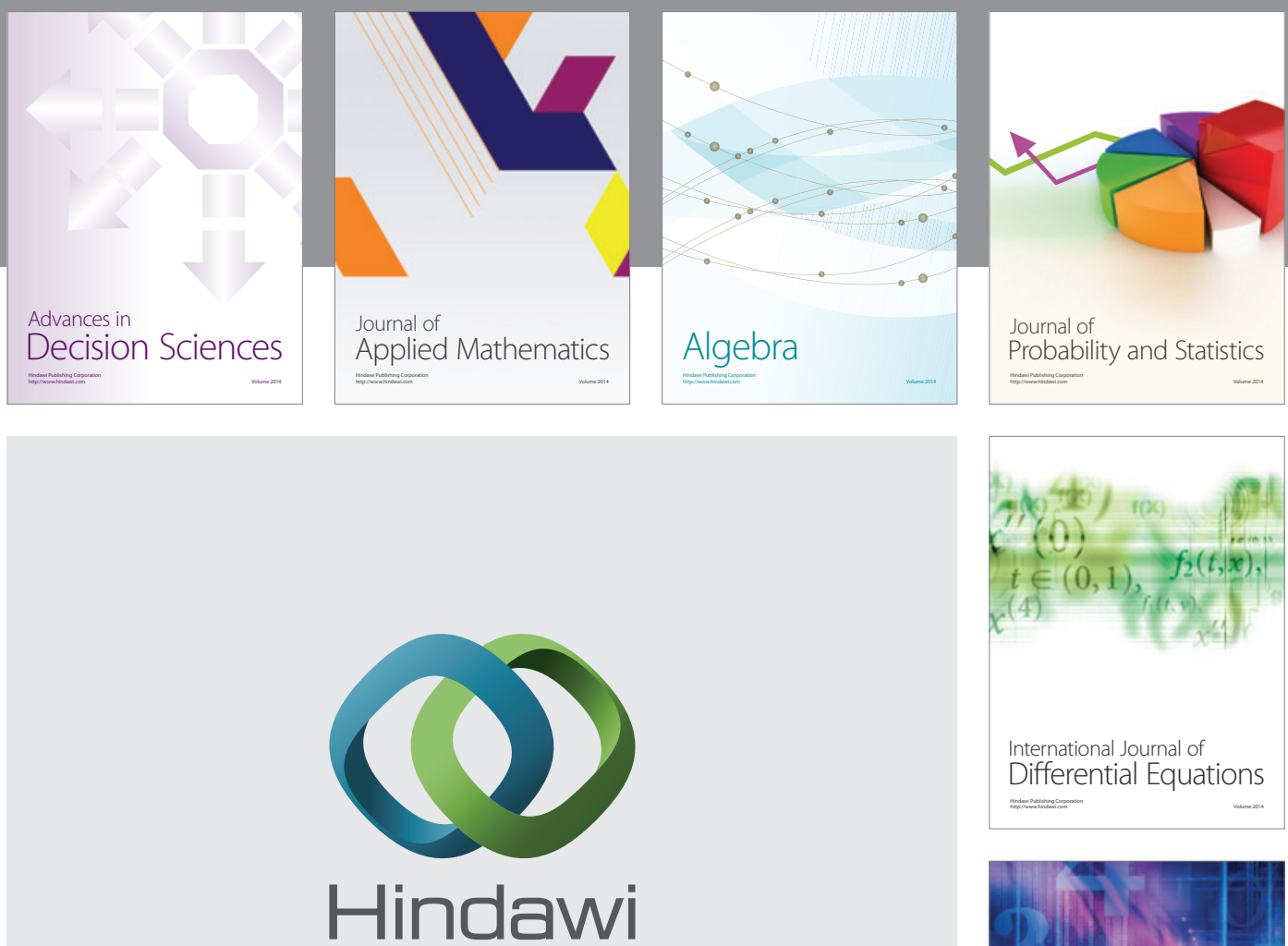

Submit your manuscripts at http://www.hindawi.com
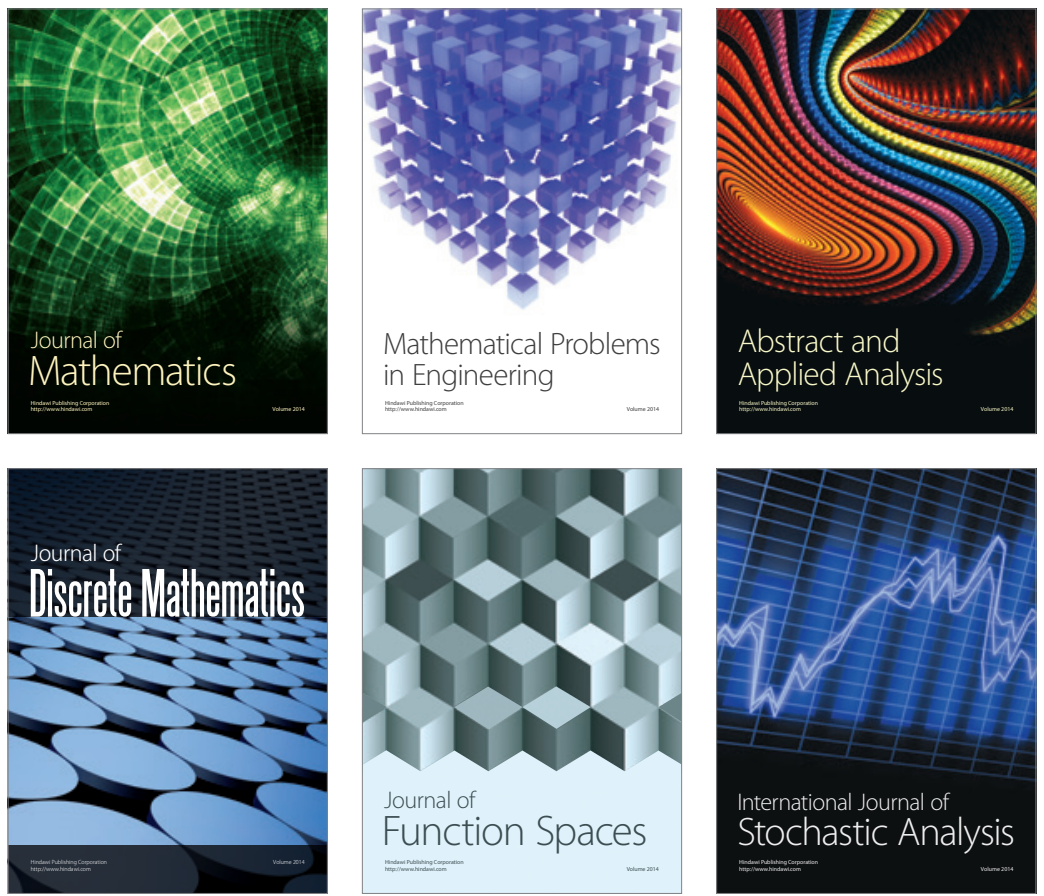

Journal of

Function Spaces

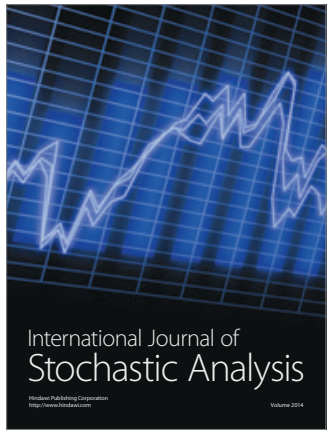

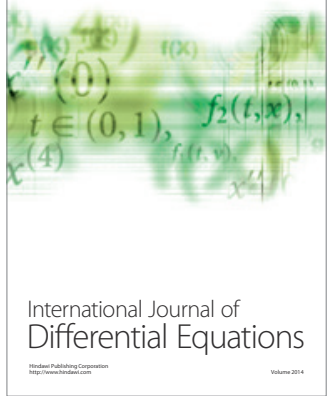
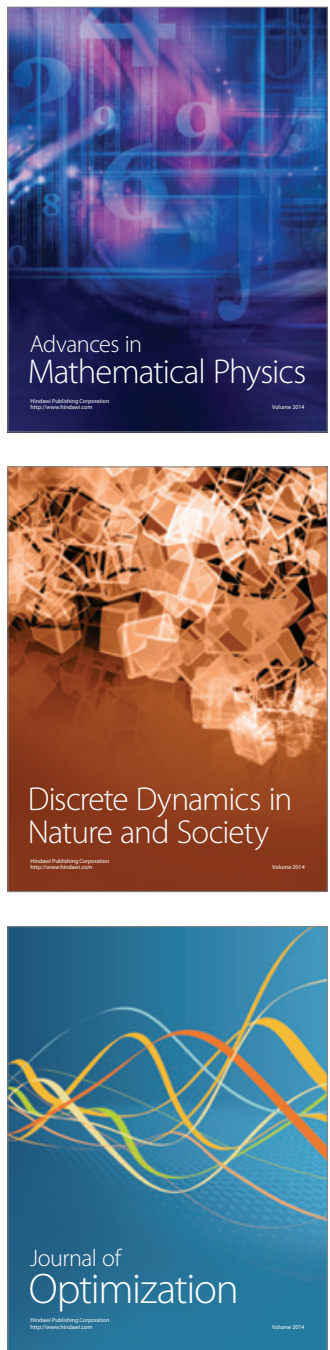